\title{
Recent Results From the T2K Experiment
}

\author{
Jeremy P. Lopez, For the T2K Collaboration \\ University of Colorado Boulder \\ E-mail: jeremy. lopez@colorado.edu
}

The T2K (Tokai to Kamioka) experiment is a long-baseline neutrino oscillation experiment using the neutrino beamline at the J-PARC facility in Japan. T2K measures the neutrino beam at two near detectors located $280 \mathrm{~m}$ from the target and again at Super-Kamiokande, $295 \mathrm{~km}$ away.With measurements of the oscillated neutrinos at Super-Kamiokande and constraints from the near detectors, $\mathrm{T} 2 \mathrm{~K}$ is able to provide high precision measurements of neutrino oscillation parameters. T2K provided the first evidence for a non-zero value of the mixing angle $\theta_{13}$ and has continued improving its results with a joint fit to muon neutrino disappearance and electron neutrino appearance samples. In 2015, T2K also released its first result on antineutrino oscillations. This work provides a summary of recent results in neutrino oscillation physics from $\mathrm{T} 2 \mathrm{~K}$ and also briefly discusses some of the other work done by $\mathrm{T} 2 \mathrm{~K}$.

XIII International Conference on Heavy Quarks and Leptons

22-27 May, 2016

Blacksburg, Virginia, USA

${ }^{*}$ Speaker. 


\section{Introduction}

The three known types of neutrinos can be described as flavor eigenstates, $v_{\alpha}$, or as mass eigenstates, $v_{i}$. These two bases are related via a unitary transformation,

$$
\left(\begin{array}{c}
v_{e} \\
v_{\mu} \\
v_{\tau}
\end{array}\right)=\left(\begin{array}{ccc}
U_{e 1} & U_{e 2} & U_{e 3} \\
U_{\mu 1} & U_{\mu 2} & U_{\mu 3} \\
U_{\tau 1} & U_{\tau 2} & U_{\tau 3}
\end{array}\right)\left(\begin{array}{c}
v_{1} \\
v_{2} \\
v_{3}
\end{array}\right)
$$

where the matrix $U$ is known as the Pontecorvo-Maki-Nakagawa-Sakata (PMNS) matrix [1]. For Dirac neutrinos, the PMNS matrix can be parameterized as a product of three rotation matrices,

$$
U=\left(\begin{array}{ccc}
1 & 0 & 0 \\
0 & c_{23} & s_{23} \\
0 & -s_{23} & c_{23}
\end{array}\right)\left(\begin{array}{ccc}
c_{13} & 0 & s_{13} e^{-i \delta} \\
0 & 1 & 0 \\
-s_{13} e^{i \delta} & 0 & c_{13}
\end{array}\right)\left(\begin{array}{ccc}
c_{12} & s_{12} & 0 \\
-s_{12} & c_{12} & 0 \\
0 & 0 & 1
\end{array}\right)
$$

where $c_{i j}=\cos \theta_{i j}$ and $s_{i j}=\sin \theta_{i j}$. The PMNS matrix has four parameters, the three mixing angles $\theta_{12}, \theta_{23}$, and $\theta_{13}$ and a complex phase $\delta$, which parameterizes charge parity (CP) violation. Using this matrix, the probability of a neutrino with energy $E$ starting in flavor $\alpha$ oscillating to flavor $\beta$ after traveling a distance $L$ can be calculated to be

$$
P\left(v_{\alpha} \rightarrow v_{\beta}\right)=\left|\sum_{i j} U_{\alpha i}^{*} U_{\beta i} U_{\alpha j} U_{\beta j}^{*} \exp \left(-i \frac{L \Delta m_{i j}^{2}}{2 E}\right)\right| .
$$

This equation gives the oscillations a characteristic shape that is a sum of sinusoidal functions of $L / E$ where the wavelengths are governed by the squared mass splittings, $\Delta m_{i j}^{2}=m_{i}^{2}-m_{j}^{2}$, between the three mass eigenstates.

In recent years, many experiments have been conducted to test this model and to make precision measurements of its parameters. These experiments use a variety of techniques to measure oscillations of beam, reactor, solar, and atmospheric neutrinos, with energies ranging from the $\mathrm{MeV}$ scale up to many $\mathrm{GeV}$. While a great deal has been measured already, there are still a number of open questions about neutrino oscillations.

So far, measurements have only yielded the magnitude of $\Delta m_{32}^{2}$, while the magnitude and sign of $\Delta m_{12}^{2}$ has been measured. As a result, there is an ambiguity in the ordering of the three mass states. $\Delta m_{32}^{2}>0$ would make $v_{1}$, which is primarily composed of the $v_{e}$ flavor state, the lightest mass state. This is known as the "normal hierarchy." $\Delta m_{32}^{2}<0$ gives the inverted hierarchy. CP violation in neutrino oscillations, which is seen in the form of differences between the oscillation probabilities of neutrinos and antineutrinos has also not yet been measured. The mixing angle $\theta_{23}$ is known to give near-maximal mixing $\left(\theta_{23} \approx 45^{\circ}\right)$, but it is not yet known if it is exactly maximal or, if not, in which octant $\theta_{23}$ lies. Finally, it is also conceivable that more neutrinos exist, and hints of this may be seen if the three neutrino mixing model is unable to explain all experimental results.

Resolving these problems requires measuring the oscillations of the different flavors of neutrinos and antineutrinos to high precision. The T2K experiment is one such experiment using a man-made beam of high energy neutrinos. 


\section{The T2K Experiment}

The $\mathrm{T} 2 \mathrm{~K}$ experiment [2] is a long-baseline neutrino oscillation experiment operating at the J-PARC facility in Tokai village in Japan's Ibaraki prefecture. The J-PARC site includes the beam facility, beam monitors and the T2K near detector complex. The T2K far detector is SuperKamiokande (SK), located in the Kamioka Observatory $295 \mathrm{~km}$ away in the mountains south of the city of Toyama.

\subsection{The T2K Beam}

$\mathrm{T} 2 \mathrm{~K}$ uses the high-intensity $30 \mathrm{GeV}$ proton beam from the J-PARC main ring accelerator. The protons are then directed into a graphite target, they produce many hadrons. The resulting charged particles are focused in a series of three magnetic horns and pass into a long decay pipe. Pions, which make up most of the charged particles, decay in flight to create a beam consisting mainly of muon neutrinos or antineutrinos, depending on the beam mode.

The Kamioka site lies $2.5^{\circ}$ away from the beam axis. An off-axis beam provides a neutrino spectrum with narrow peak centered around approximately $600 \mathrm{MeV}$, which corresponds to the energy at which the fraction of neutrinos that have oscillated away from the initial $v_{\mu}$ state is maximized. Because of the near maximal mixing of $v_{\mu}$ from $\theta_{23}$, the neutrino flux at Kamioka is composed primarily of $v_{\tau}$ with much smaller contribution from $v_{e}$ and $v_{\mu}$. This effect can be seen in Fig. 1. More information on predicting the T2K neutrino flux can be found in Ref. [3].

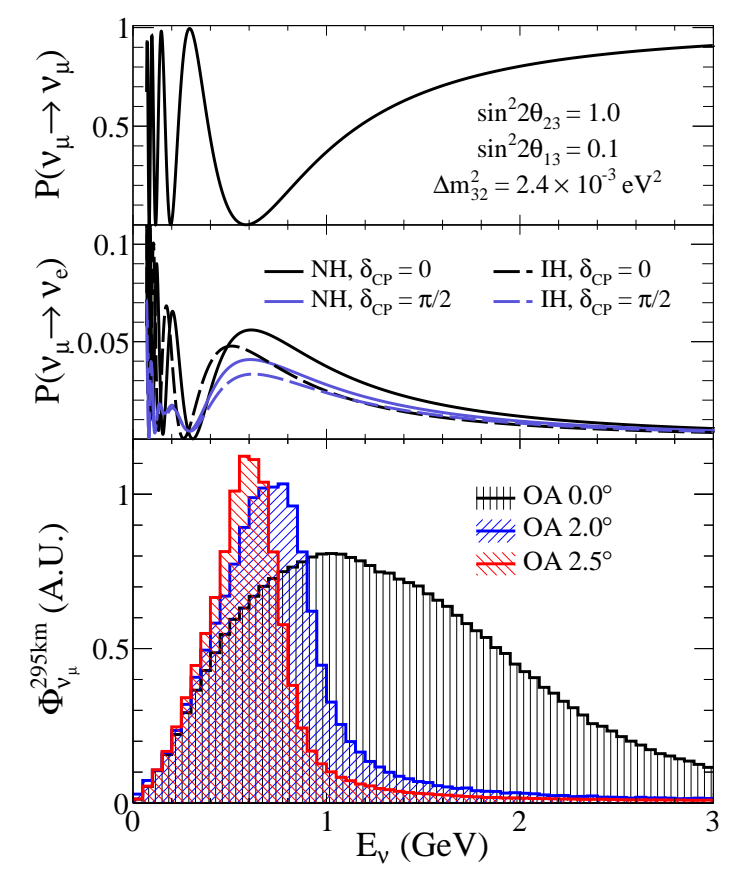

Figure 1: Top: The $v_{\mu} \rightarrow v_{\mu}$ oscillation probability as a function of neutrino energy expected at the far detector. Middle: The $v_{\mu} \rightarrow v_{e}$ probability. Bottom: The expected neutrino spectrum for several different angles with respect to the beam axis. The $2.5^{\circ}$ option shows a narrow peak centered around the oscillation maximum. 


\subsection{Far Detector: Super-Kamiokande}

Super-Kamiokande [4] measures the beam after oscillations. At the energies of interest to $\mathrm{T} 2 \mathrm{~K}$, near the $600 \mathrm{MeV}$ peak in the neutrino spectrum, there is not enough energy to produce $v_{\tau}$ charged current (CC) events. As a result, SK looks for two types of oscillations: (1) muon neutrino disappearance, by counting the deficit of $v_{\mu}$ charged current events seen at SK compared to predictions with no oscillations, and (2) electron neutrino appearance by counting the excess of $v_{e}$ charged current events compared to predictions. T2K used an early measurement of electron neutrino appearance to obtain the first evidence for the existence of a non-zero value of $\theta_{13}$. SK is a large water Cherenkov detector and is able to distinguish $v_{\mu}$ events from $v_{e}$ events using the event topology. In $v_{\mu}$ events, the muon loses energy steadily as it stops, resulting in clearly defined rings of Cherenkov radiation. In contrast, electrons from $v_{e}$ events undergo a great deal of multiple scattering, resulting in rings of Cherenkov radiation with edges that are much less sharp.

\subsection{Near Detectors}

High precision measurements require detailed knowledge of the neutrino flux and interaction cross sections in order to make accurate predictions for what is expected to be measured at the far detector. T2K uses external hadron production measurements from NA61/SHINE and external cross section measurements to inform the flux and interaction models. In order to further constrain the physics models using data from the J-PARC beam, T2K uses two near detectors, located approximately $280 \mathrm{~m}$ from the target.

INGRID is an on-axis detector consisting of 16 modules with alternating layers of plastic scintillator bars and iron. It monitors the health of the beam by measuring properties such as event rates, the beam direction, and the beam profile [5]. An additional module consisting only of plastic scintillator layers allows for the measurement of neutrino cross sections on scintillator. Figure 2 shows a drawing of the INGRID detector.

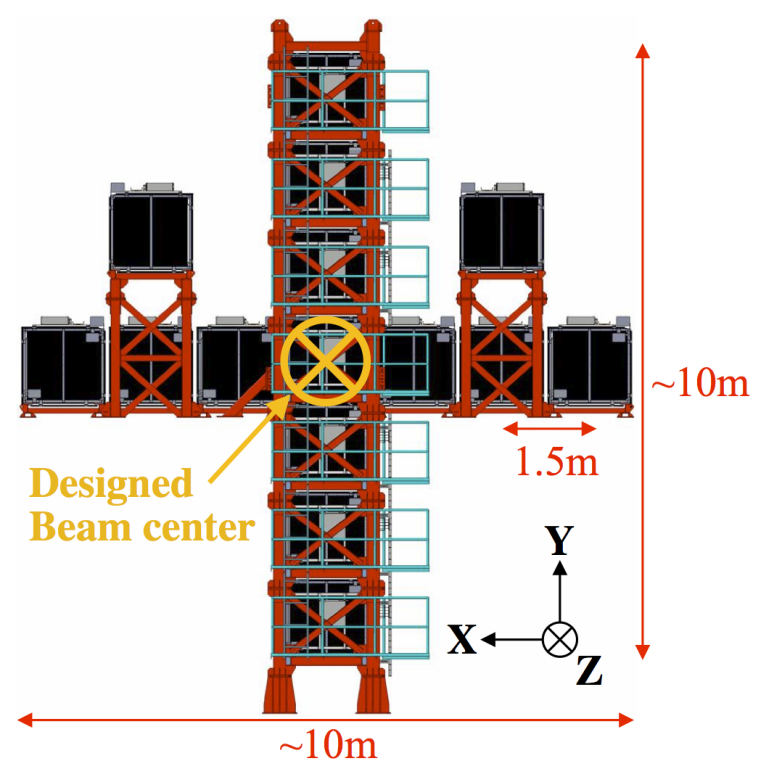

Figure 2: Schematic of the INGRID detector. From [2]. 
ND280 is the off-axis near detector. It is located $2.5^{\circ}$ away from the beam axis and measures the unoscillated neutrino spectrum that is seen at SK. ND280 is a magnetized tracking detector using various subdetectors, including time projection chambers (TPCs) [6], scintillator-based finegrained trackers (FGDs) [7], electromagnetic calorimeters (ECals) [8], and a $\pi^{0}$ detector (P0D) [9], as well as muon detectors (SMRDs) [10]. The various subdetectors of ND280, with the exception of the muon range detectors, are held within the former UA1 electromagnet, which provides a 0.2 $\mathrm{T}$ magnetic field. The curvature of tracks in the magnetic field allows for precise measurements of the charge and momentum of charged particles within the detector. Figure 3 shows how the different components of ND280 fit together.

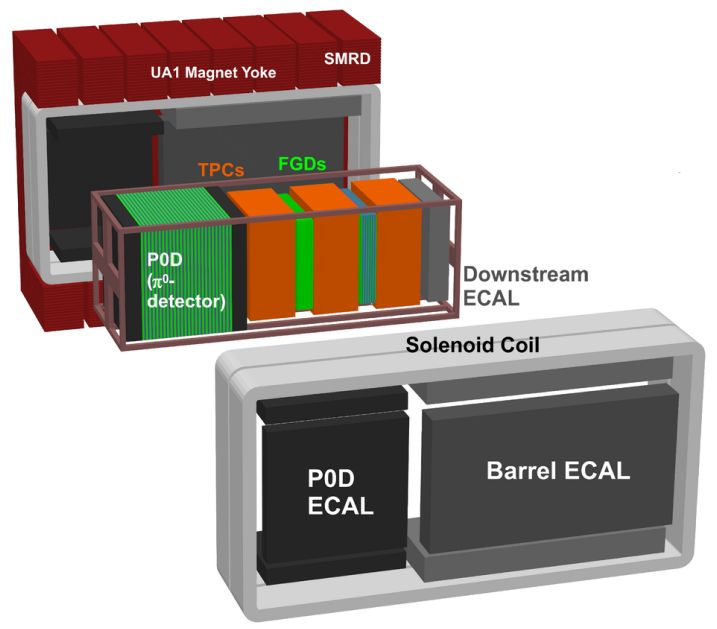

Figure 3: Exploded view of the ND280 detector. Not shown are the side muon range detectors, which are placed within the magnet yoke. From [2].

\section{Oscillation Results}

\subsection{Joint $v_{\mu}, v_{e}$ fit}

The most recent published T2K fit to neutrino oscillation parameters used $6.57 \times 10^{20}$ protons on target (POT) of data taken between 2010 and 2013. After this period, T2K has collected mostly antineutrino data. This analysis is described in detail in Ref. [11]. The near detector flux and interaction constraints are obtained by defining three samples of data based on reconstructed final state topology: charged current with no pions $(\mathrm{CC} 0 \pi)$, charged current with $\pi^{+}$production $\left(\mathrm{CC} 1 \pi^{+}\right)$, and all other charged current events (CCOther). These selections use events occurring in the scintillator-based FGDs and passing into the TPCs. A maximum likelihood fit is applied to the muon momentum and angle with respect to the beam, $\left(p_{\mu}, \cos \theta_{\mu}\right)$, distributions of these samples. The likelihood fit considers the uncertainties of the input model, which includes parameters for the neutrino flux, various types of interactions, and ND280 detector uncertainties. Figure 4 shows the effect of the ND280 fits on the predictions of the SK event rates. The ND280 constraint reduces the uncertainty by approximately a factor of three. 

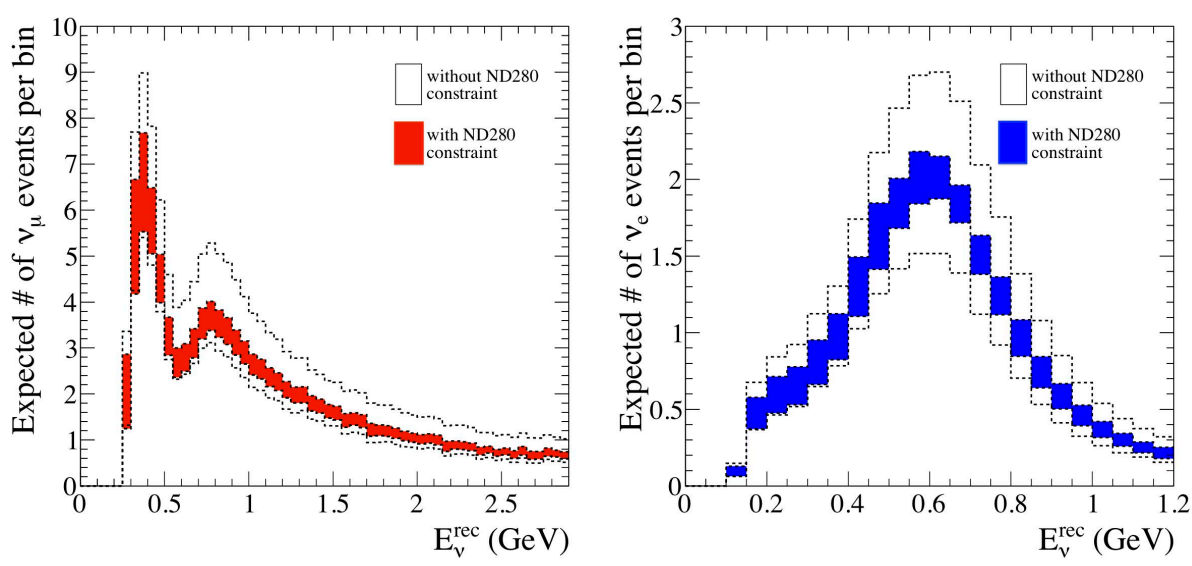

Figure 4: Effect of the ND280 constraint on the predictions for SK samples for typical oscillation parameters. The input (unconstrained) model is shown in the dashed line, and the constrained model is given in the solid models. Left: SK $v_{\mu}$ selection. Right: SK $v_{e}$ selection. Plots from [11].

The SK data analysis uses two selections - CCQE-like $v_{\mu}$ events and CCQE-like $v_{e}$ events. Figure 5 shows the distributions for both selections as well as the predicted distributions with and without oscillations. The oscillation analysis fits the oscillation parameters $\Delta m_{32}^{2}, \theta_{23}, \theta_{13}$, and $\delta$ to the SK samples while marginalizing over nuisance parameters such as the flux, cross sections, and detector uncertainties. Figure 6 shows the resulting uncertainty contours for the $\left(\Delta m_{32}^{2}, \theta_{23}\right)$ phase space. This analysis represents the most precise measurement in the $\left(\Delta m_{32}^{2}, \theta_{23}\right)$ phase space. Figure 7 shows the result for the CP violating phase $\delta$ when including constraints on $\theta_{13}$ from reactor experiments. The T2K with reactor constraint analysis excludes $\delta \in[0.15,0.83] \pi$ for the normal hierarchy and $\delta \in[-0.08,1.09] \pi$ for the inverted hierarchy.
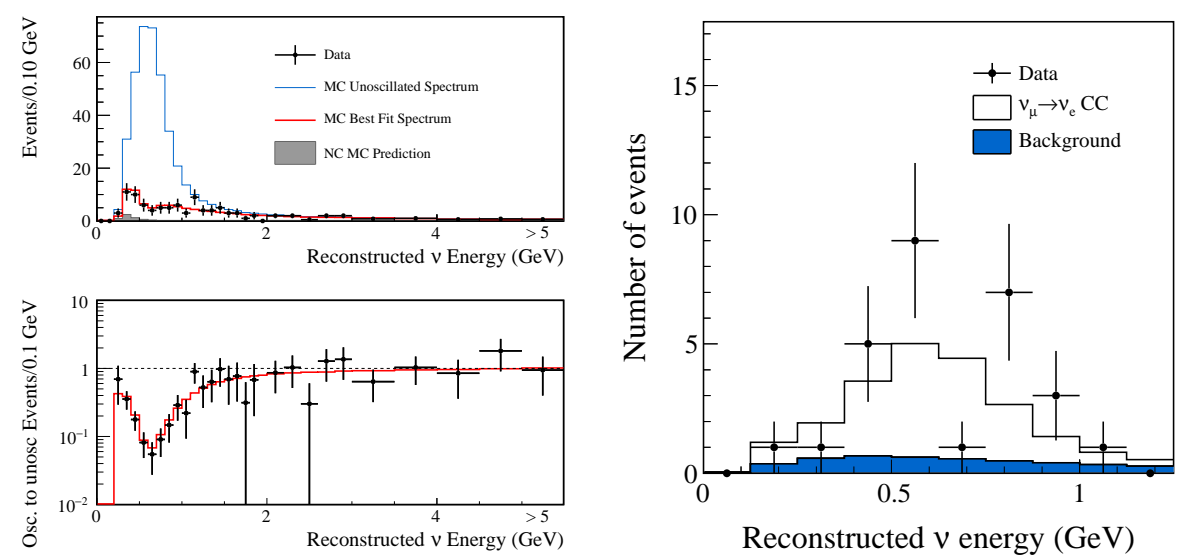

Figure 5: Data samples measured by SK. The black points are the data and the blue represents what would be expected if there were no oscillations. Left: $v_{\mu}$ disappearance sample. The red curve is the best fit spectrum. Right: $v_{e}$ appearance data. The solid curve is the appearance signal for some typical values of oscillation parameters. Plots from [11]. 

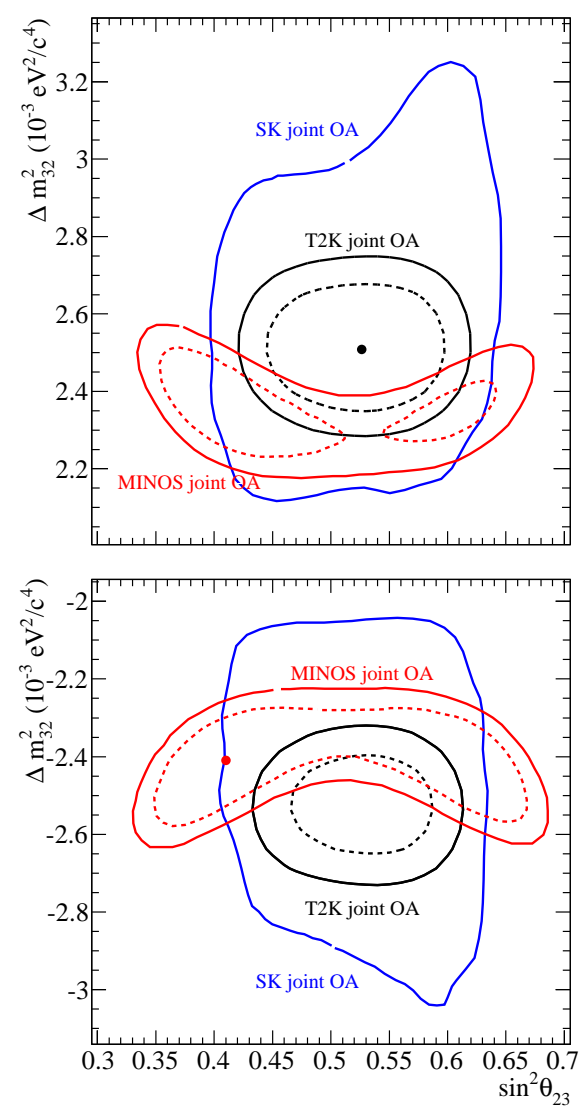

Figure 6: Results for $\Delta m_{32}^{2}$ and $\sin ^{2} \theta_{23}$ from a frequentist analysis of $v_{\mu}$ disappearance and $v_{e}$ appearance data with $68 \%$ (dashed) and $90 \%$ (solid) confidence regions. The T2K result is the most precise so far for both the normal hierarchy (top) and the inverted hierarchy (bottom). From [11].

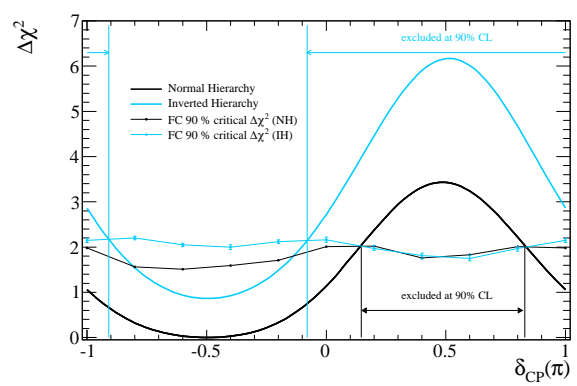

Figure 7: $\Delta \chi^{2}$ as a function of $\delta_{C P}$ from the T2K fit using constraints from reactor experiments for $\theta_{13}$. T2K is able to rule out parts of the $\delta_{C P}$ phase space at $90 \%$ confidence level. From [11]. 


\subsection{First Antineutrino Measurements}

T2K has also published its first antineutrino results, looking at $\bar{v}_{\mu} \rightarrow \bar{v}_{\mu}$. The analysis, described in Ref. [12], proceeds similarly to the neutrino analysis, but with a fit to a two-neutrino model rather than the joint fit. The near detector constraint in the $\bar{v}_{\mu}$ fit uses four ND280 samples: $\bar{v}_{\mu}$ and $v_{\mu}$ charged current with 1 track and $\bar{v}_{\mu}$ and $v_{\mu}$ charged current with $>1$ track. The ND280 data used includes $5.82 \times 10^{20}$ POT of neutrino mode data and $0.43 \times 10^{20}$ POT of antineutrino mode data, while the SK sample used $4.01 \times 10^{20}$ POT of antineutrino mode data.

The oscillation fit gives the result seen in Figure 8. The fit values are in good agreement with other results and also with the $\mathrm{T} 2 \mathrm{~K}$ neutrino mode parameters.

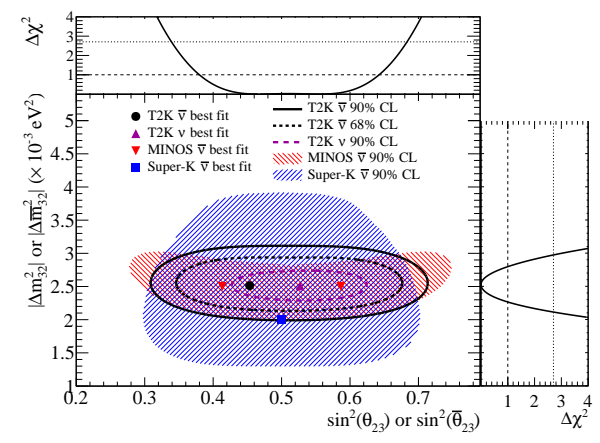

Figure 8: Result of the $\bar{v}_{\mu} \rightarrow \bar{v}_{\mu}$ analysis with 68\% (dashed) and $90 \%$ (solid) confidence regions. From [12].

T2K has also looked for appearance of electron antineutrinos. In the first such search by T2K, three candidate $\bar{v}_{e}$ events are found. Given the neutrino oscillation parameters measured by T2K, it is estimated that approximately 3 signal events would be expected, with a background of about 1.5 , with the exact values depending on the true value of the CP violating phase $\delta$. These data are consistent with both the background alone and with the background+signal model. Thus, more data is required in order to make a definitive measurement of $\bar{v}_{e}$ appearance.

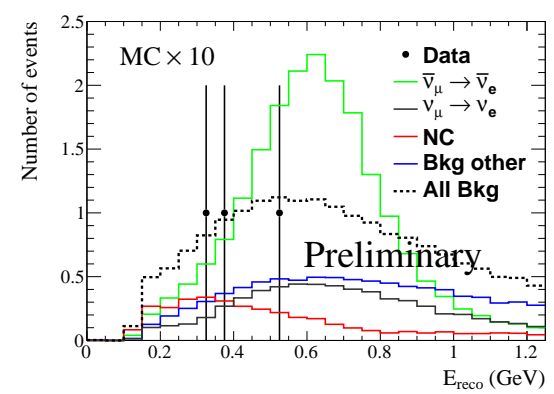

Figure 9: Preliminary results for the $\bar{v}_{e}$ events with expected background and signal distributions.

\section{Neutrino Interactions}

The T2K physics program includes more than just oscillation measurements. The near detectors also produce measurements of neutrino cross sections. One recent analysis is a measurement 
with ND280 of the charged current $v_{\mu}$ cross section on scintillator with no pions in the final state [13]. This measurement used two different selections. One looks for a muon with no or one reconstructed proton and no other tracks and extracts the cross section with a binned likelihood fit. The other looks for a muon with no pion candidate tracks and extracts the cross section using Bayesian unfolding method [14]. The systematics in these results are dominated by the flux uncertainty. The cross section as a function of muon momentum for several ranges of muon angles are given in Figure 10.
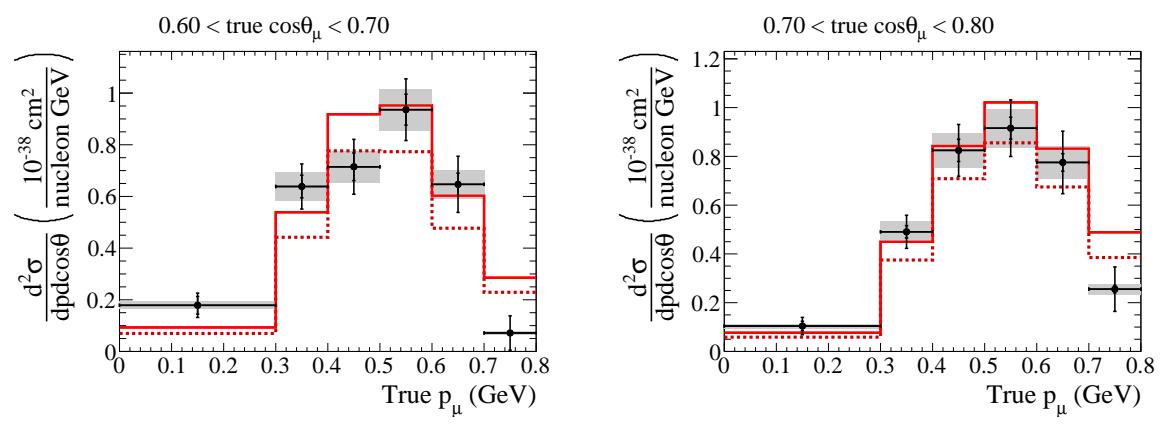

Figure 10: The $\mathrm{CC} 0 \pi$ cross section as a function of muon momentum for several ranges of muon angles. The black points represent the data, with the error bars showing the shape uncertainties and the gray bands showing the normalization-only uncertainty. The red curves are predictions from Nieves (red dotted line) $[15,16]$ and Martini (red solid line)[17, 18]. Plots from [13].

\section{Conclusions}

The T2K experiment has now measured the oscillations of both muon neutrinos and muon antineutrinos and has produced leading measurements of oscillations parameters using $v_{\mu}$ disappearance, $v_{e}$ appearance, and $\bar{v}_{\mu}$ disappearance samples. T2K has also searched for electron antineutrino appearance and is producing many measurements of neutrino interactions. T2K has only taken $\sim 20 \%$ of its expected beam and will continue to take data and improve these results.

\section{References}

[1] K.A. Olive et al. (PDG), Chin. Phys. C38, 090001 (2014).

[2] K. Abe et al., Nucl. Instrum. Meth. A659, 106-135 (2011).

[3] K. Abe et al., Phys. Rev. D87, 012001 (2013).

[4] S. Fukuda et al., Nucl. Instrum. Meth. A501 418-462 (2003).

[5] K. Abe et al., Nucl. Instrum. Meth. A694 211-223 (2012).

[6] N. Abgrall et al., Nucl. Instrum. Meth. A637 25-46 (2011).

[7] P.-A. Amaudruz et al., Nucl. Instrum. Meth. A696 1-31 (2012).

[8] D. Allan et al., JINST 8, P10019 (2013).

[9] S. Assylbekov et al., Nucl. Instrum. Meth. A686 48-63 (2012). 
[10] S. Aoki et al., Nucl. Instrum. Meth. A698 135-146 (2013).

[11] K. Abe et al., Phys. Rev. D 91, 072010 (2015).

[12] K. Abe et al., Phys. Rev. Lett. 116, 181801 (2016).

[13] K. Abe et al., Phys. Rev. D 93, 112012 (2016).

[14] G. D’Agostini, Nucl. Instrum. Meth. A362, 487-498 (1995).

[15] J. Nieves, I.R. Sim, and M.V. Vacas, Phys. Lett. B 707, 72 (2012).

[16] J. Nieves et al., Phys. Rev. D 85, 113008 (2012).

[17] M. Martini et al., Phys. Rev. C 80, 065501 (2009).

[18] M. Martini and M. Ericson, Phys. Rev. C 90, 025501 (2014). 